THE KURUME MEDICAL JOURNAL

Vol. 36, p. 35-39, 1989

\title{
Transgastric Vagotomy with Selective Proximal Vagotomy for Duodenal Ulcer
}

\author{
JINRYO TAKEDA, YUH HIRAI, HIROSHI FUJIMASA, KEN HASHIMOTO \\ AND TERUO KAKEGAWA \\ Department of Surgery, Kurume University School of Medicine, Kurume, 830 Japan
}

\author{
Received for publication February 17, 1989
}

\begin{abstract}
Summary: To improve surgical results of duodenal ulcer, transgastric myotomy (TGM) was added to the traditional selective proximal vagotomy (SPV) and its efficacy was evaluated clinically in 40 patients. 35 men and 5 women were involved, with a mean age of $33.07 \pm 14.25$ years. Pyloroplasty was added in 12 operations for stenosis and perforation. 28 patients in this series underwent TGM with SPV without drainage. In the 36 patients, basal and maximal acid output (BAO and MAO) was compared preoperatively and at 6 months postoperatively. A satisfactory reduction 'of acid output was achieved, with a mean reduction rate of $71.4 \%$ in $\mathrm{BAO}_{\text {ind }}$ and $79.9 \%$ in MAO. All 40 patients were negative in Hollander's insulin stimulation test at 6 months postoperatively. The gastric mucosa was injured during myotomy in 2 of the patients (5.0\%), and was simply sutured using 3-0 Dexon, without causing any problems. No other early or late postoperative complication was present. In addition, no peptic ulcer recurrence has been noted over a maximal follow-up of 8 years. The present results suggest the completeness of gastric denervation by TGM $+\mathrm{SPV}$, and establish the efficacy of TGM with SPV, and therefore this method is recommended in the surgical treatment of duodenal ulcer.
\end{abstract}

Key words: duodenal ulcer - transgastric myotomy - selective proximal vagotomy - acid output - ulcer recurrence

\section{Introduction}

The most important aims of surgical treatment for duodenal ulcer are reduction of gastric acid output and the preservation of the gastric antral function. Despite the large variety of operative procedures, ulcer surgery is still a controversial issue; selective proximal vagotomy (SPV) being a more recent innovation in this field. The risk of recurrence is almost the only disadvantage of SPV, and this operation is still associated with a recurrence rate of 6 to $15 \%$. One of the causes for such recurrence is the incompleteness in SPV.

For further improvement in operative results in duodenal ulcer, Transgastric Myotomy (TGM) was added to the traditional SPV to ensure completeness of denervation, and the combined efficacy was investigated in 40 patients.

\section{Operative Techniques}

After traditional SPV was completed, TGM was performed. The abdominal esophagus was dissected to within $2 \mathrm{~cm}$ from the esophago-gastric (E-G) junction. Gastric bow-shaped myotomy was carried out from the lesser curvature side of the E-G junction through the inferior pole of 
the spleen on the greater curvature side, with care taken to avoid injury to the spleen and the short gastric artery. Myotomy was then performed on both the anterior and the posterior walls of the stomach. During myotomy, the submucosa should be fully exposed for complete denervation of the serosa and the muscle layer of the stomach.

Following myotomy, sero-serosal suture was carried out after inversion of the serosa and the muscle projecting the cut surface of the stomach (Fig. 1).

\section{Materials and Methods}

During the 8 years from 1980 to 1987 , we have performed SPV + TGM in 40 patients with duodenal ulcer. 35 men and 5 women were involved with an age range from 14 years to 63 years (ave. 33.07士 14.25). Indications for operation, besides intractability, included duodenal stenosis in $36(90 \%)$ patients and duodenal perforation in $4(10 \%)$ patients. Pyloroplasty was added in 12 operations for stenosis and perforation, including 2 Heineke Mikulitz, 7 Holle, 1 Jabouly, and 2 Finney methods. 28 patients in this series underwent SPV + TGM without drainage (Table 1).

\section{Results}

\section{Gastric Acid Output}

In the 36 patients with duodenal ulcer, excluding the 4 emergency cases, the basal and maximal acid outputs (BAO and MAO) were compared preoperatively and at 6 months postoperatively. A satisfactory reduction in acid output was achieved, with a mean reduction rate of $71.4 \%$ in BAO and $79.9 \%$ in MAO (Table 2).

All 40 patients were negative in Hollander's insulin stimulation test at 6 months postoperatively.

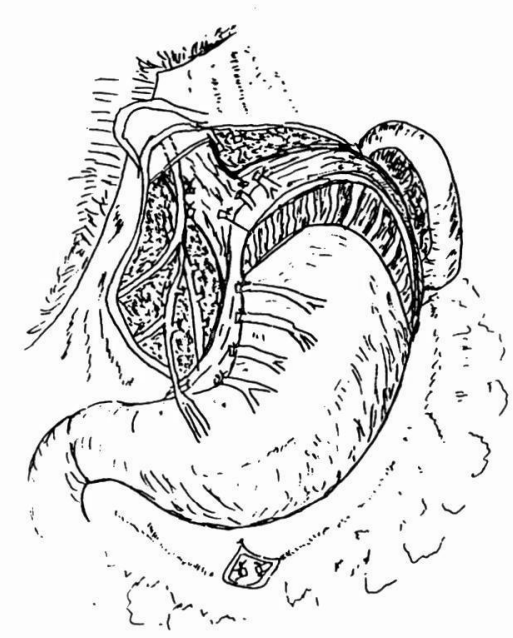

Fig. 1. TGM with SPV: Showing bow-shaped seromyotomy at the anterior gastric wall after traditional SPV. Right epigastric branches were divided and cut not within $7 \mathrm{~cm}$ from the pyloric ring.

TABLE 1

Clinical profile of the 40 patients

$\begin{array}{lr}\text { Sex Male: } 35 & \text { Female: } 5 \\ \text { Age }(\mathrm{M} \pm \mathrm{SD}) / \text { years } & 33.07 \pm 14.25\end{array}$

Indication for Operation

Intractable(incluing stenosis) $\quad 36$

Perforation

4

Additional Pyloroplasty

Heineke-Mikulicz 2

Holle 7

Jabouly 1

Finney 2

\section{Serum Gastrin Levels}

In the group of pyloroplasty (-), preoperative fasting serum gastrin was 71.9 $\pm 13.5 \mathrm{pg} / \mathrm{ml}$, and $116.8 \pm 62.4 \mathrm{pg} / \mathrm{ml}$ after eating beaf extract preoperatively. In the group of pyloroplasty $(+)$, preoperative fasting serum gastrin was $54.6 \pm 5.8 \mathrm{pg} / \mathrm{ml}$, and $135.8 \pm 9.2 \mathrm{pg} / \mathrm{ml}$ after eating beaf extract. At 6 months postoperatively, in the 
TABLE 2

Gastric Acid Output Reduction Rate after SPV with TGM

\begin{tabular}{c|c|c|c}
\hline $\begin{array}{c}\text { Acid Output } \\
(\mathrm{mEq} / \mathrm{h})\end{array}$ & Pre-Op. & $\begin{array}{c}\text { Post-Op. } \\
(6 \text { Months })\end{array}$ & $\begin{array}{c}\text { Reduction } \\
\text { Rate (\%) }\end{array}$ \\
\hline BAO & 5.51 & 1.57 & 71.4 \\
MAO & 12.74 & 2.52 & 79.9 \\
\hline
\end{tabular}

TABLE 3

Serum Gastrin Levels: Variation across eating ( $p g / m l)$

\begin{tabular}{c|c|c|c|c}
\hline \multirow{2}{*}{ Pyloroplasty } & \multicolumn{2}{|c|}{ Pre-Op. } & Post-Op. (6 Months) \\
\cline { 2 - 5 } & fasting & beef extract & fasting & beef extract \\
\hline $\begin{array}{c}\text { Negative (-) } \\
\mathrm{n}=17\end{array}$ & $71.9 \pm 13.5$ & $116.9 \pm 62.4$ & $71.9 \pm 34.5$ & $205.0 \pm 72.9$ \\
$\begin{array}{c}\text { Positive (+) } \\
\mathrm{n}=8\end{array}$ & $54.6 \pm 5.8$ & $135.8 \pm 9.2$ & $97.0 \pm 18.8$ & $258.3 \pm 34.9$ \\
\hline
\end{tabular}

group of pyloroplasty (-), fasting serum gastrin was $71.9 \pm 34.5 \mathrm{pg} / \mathrm{ml}$, and peak serum gastrin was $205.6 \pm 72.6 \mathrm{pg} / \mathrm{ml}$ after eating beaf extract. In the group of pyloroplasty $(+)$, fasting serum gastrin was $97.0 \pm 18.8 \mathrm{pg} / \mathrm{ml}$, and peak serum gastrin was $258.3 \pm 34.9 \mathrm{pg} / \mathrm{ml}$ after eating beaf extract (Table 3 ).

\section{Mortality and Morbidity}

The gastric mucosa-was injured during myotomy in 2 of 40 patients $(5.0 \%)$. The injured mucosa was simply sutured by 3-0 Dexon, and did not cause any problem. No other early or late post operative complication was present. In addition, no peptic ulcer recurrence has been noted.

\section{Discussion}

Selective proximal vagotomy (SPV), selectively denervates only the proximal acid producing portion of the stomach. Innervation of the antrum is retained and thus the mortility of this part of the stomach is undisturbed. The advantages of SPV are fully realized only when py- loroplasty is not performed. A pyloroplasty should be performed only for cicatricial gastric exit obstruction.

The particular advantages of SPV are low risk for complication preservation of the gastric antral function and the performance of the whole stomach, and wide spread absence of any long-term side effect, without ulcer recurrence. Johnston (1975) reported only 17 deaths following 5539 operations carried out in Britain, Scandinavia, and the United States; an operative mortality of $0.3 \%$. The risk of recurrence is almost the only disadvantage of SPV, and is still associated with a recurrence rate of 6 to $15 \%$. One of the causes of such recurrence is the incompleteness of SPV. In duodenal ulcers, the possible causes for a recurrence of SPV was demonstrated by Holle et al. (1988). $79 \%$ of the recurrence were due to technical error.

For further improvement in operative results in duodenal ulcer, Transgastric Myotomy (TGM) was added to the traditional SPV to achieve complete denervation by the Petropoulos method (1979). Vagotomy combined with antrectomy (40\% 
gastrectomy) has the lowest ulcer recurrence rate and also achieves the greatest acid reduction, both basal and stimulated. However, morbidity and mortality are greater with vagotomy procedures combined with gastric resection than vagotomy without gastric resection. If gastrectomy is not included, the greatest acid reduction is attained by successful SPV, followed by Selective Gastric Vagotomy (SGV), and finally by Trancal Vagotomy (TV).

The need for a careful dissection encircling the intra-abdominal esophagus for at least $6 \mathrm{~cm}$ has been indicated by Kronborg et al. (1977) and Nyhus et al. (1980) for complete SPV. In TGM with $\mathrm{SPV}$, the abdominal esophagus is dissected only up to $2 \mathrm{~cm}$ from the E-G junction to avoid esophageal injury and postoperative achalasialike syndrome. The esophageal circular myotomy recommended by Hedenstedt et al. (1980) allows for safe severing of the intramural vagus branches, but must be viewed skeptically because of the possible postoperative complications. Similar objections have been reported by Taylor (1980) and Chen et al. (1983), involving the lesser gastric curvature and fundus myotomy, with the extent of dissection disputed at the crow's food area.

The effectiveness of vagotomy has been predominantly studied in patients with duodenal ulcer, since the pathogenetic connection with acid secretion is more convincing for duodenal ulcer than for gastric ulcer. There is a close correlation between the frequency of ulcer recurrence and the extent of acid reduction achieved by vagotomy. A satisfactory reduction of acid output was achieved and a mean reduction rate of $71.4 \%$ in $\mathrm{BAO}$ and $79.9 \%$ in MAO in our clinical studies of TGM with SPV.

In most patients with duodenal ulcers, the serum gastrin determination were within the normal range. The levels of gastrin released by food were increased after TGM with SPV.
After vagotomy, the G-cell population was increased in the antrum. Moreover, the amount of gastrin in the antrum mucosa was increased. In our clinical studies, the increase of the serum gastrin was within normal levels even after stimulation.

We believe that SPV + TGM is a suitable basic operation for patients with duodenal ulcer disease, without gastrinoma or antral G-cell hyperfunction whatever the serum gastrin levels.

It has been the prevailing impression that most postoperative recurrent ulcers occurred within the first 2 years after the operation.

Reinnervation of the stomach by budding of the vagal fibers has been advanced as the long term cause of recurrence. However, at this time, it would appear to occupy a minor position among the causes of a recurrence. The operative technique of SPV with TGM, is safe and simple in performance and provides excellent reduction of gastric acid secretion. The present results suggest the completeness of gastric denervation by SPV + TGM, and establish the efficacy of SPV with TGM, and therefore this method is recommended in the surgical treatment of duodenal ulcer. SPV + TGM is an ideal procedure in the surgical treatment for duodenal ulcer.

\section{References}

Busman, D. C., Volories, 'A. and Munting, D.K. (1988). Recurence rate after highly selective vagotomy. World J. Surg. 12, 217-223.

Chen, H., Chung, C. and Wu, T. (1983). Lesser gastric curve and fundus myotomy: Experimental and clinical studies. Arch. Surg. 118, 88-92.

Donahue, P. E., Yoshida, J., Richter, H. M., Liu, K., Bomвecк, T. and Nyhus, L. M. (1988). Proximal gastric vagotomy with drainage for obstructing duodenal ulcer. Surg. 104, 757-763. Hendenstedt, S., Schayan, M. and Moberg, S. 
(1980). Selective proximal vagotomy without drainage in the treatment of duodenal ulcer. The results after a standardization of the surgical technique. Acta Chir. Scand. 146, 31-34.

Holle, G. E., Frey, K. W., Thime, C. and Holle, F. K. (1988). Recurrence of peptic ulcer after selective proximal vagotomy and pyloroplasty in relation to changes in clinical sign and symptoms between 1969 and 1983. Surg. Gynecol. Obstet. 16, 271-281.

Johnston, D. (1975). Operative mortality and post-operative morbidity of highly selective vagotomy. $\mathrm{Br}$. Med. J. 4, 545-547.

KRONBORG, O. and JoRGENSEN, P. M. (1978). Influ- ence of different techniques of PGV upon the risk of recurrent DU and gastric acid secretion. Acta Chir. Scand. 143, 53-56.

Nyhus, L. M., Donahue, P.E., Trystosek, R. J., PeARL, R. K. and Вомвеск, T. (1980). Complete Vagotomy. The evolution of an effective technique. Arch. Surg. 115, 264-268.

Peteropoulos, P.C. (1979). Transgastric highly selective vagotomy (HSTRV) without drainage. Preliminary report of a new simplified procedure of treatment of duodenal ulcer. Langenbecks Arch. Chir. 350, 95-101.

TAYLER, T.V. (1979). Lesser curve superficial seromyotomy-an operation for chronic duodenal ulcer. Br. J. Surg. 66, 733-737. 